\title{
Strates
}

STRATES Matériaux pour la recherche en sciences sociales

Hors-série | 2002

Parcours dans la recherche urbaine, Michel Rochefort, un géographe engagé

\section{Michel Rochefort et la Méditerranée}

Dominique Rivière

\section{(2) OpenEdition \\ Journals}

Édition électronique

URL : http://journals.openedition.org/strates/566

ISSN : 1777-5442

\section{Éditeur}

Laboratoire Ladyss

Édition imprimée

Date de publication : 1 janvier 2002

ISSN : 0768-8067

\section{Référence électronique}

Dominique Rivière, « Michel Rochefort et la Méditerranée », Strates [En ligne], Hors-série | 2002, mis en ligne le 18 mai 2005, consulté le 07 septembre 2020. URL : http://journals.openedition.org/strates/ 566

Ce document a été généré automatiquement le 7 septembre 2020

Tous droits réservés 


\title{
Michel Rochefort et la Méditerranée
}

\author{
Dominique Rivière
}

1 Tu as depuis longtemps une activité importante dans ce qu'on peut appeler le « partenariat » ou la coopération universitaire entre la France et la rive sud de la Méditerranée. À ton avis, quel est ou quel peut être le rôle de ces échanges universitaires dans le processus de développement, et comment se sont-ils insérés dans les rapports post-coloniaux?

2 Pour moi, les échanges internationaux entre la France et les pays du Maghreb se partagent en deux groupes d'universitaires bien différenciés. Un petit nombre, dans lequel je me classe, a toujours eu une idée de participation à la décolonisation, ou au moins à l'effacement des conséquences négatives de la colonisation. D'autres se placent encore dans un « rôle directeur » de représentation de la «culture française ». Donc, de ce point de vue, je ne suis pas original quoique je me situe dans un groupe plutôt minoritaire. J'ai travaillé au Maroc, en Tunisie et en Algérie à la «formation des formateurs", en me situant comme enseignant et/ou directeur de recherches provisoire, c'est-à-dire visant à remplir une case que les intellectuels du pays ne pouvaient, ne peuvent pas encore remplir, mais en les aidant à compléter leur formation pour qu'ils puissent à terme me remplacer. Il y a eu trois phases, en particulier en Tunisie, où cette collaboration a été la plus aboutie :

3 - une première phase qui suit directement l'indépendance. Dans cette première décennie, j'avais trois missions : faire des conférences-débats sur les problèmes urbains, ce qui constituait ma spécialité - ces conférences s'adressaient davantage aux enseignants qu'aux étudiants; encadrer des examens de licence, en somme me porter caution au département de Géographie de l'université de Tunis; et enfin le plus important : assurer l'encadrement de thèses de Tunisiens ou de Français travaillant sur la Tunisie, portant toujours sur l'urbain. Il existait déjà tout un corps d'assistants non docteurs d'État, comme M'Zabi qui une fois docteur est devenu directeur du département de Géographie ou côté français Signoles qui est devenu un spécialiste de la Tunisie. J'ai fait le même travail au Maroc mais sur une durée plus longue et avec un contenu limité à la seule direction de thèses, par exemple celle de Refass sur le réseau urbain de la péninsule de Tanger, qui lui a permis de devenir professeur à l'université de Casablanca, ou plus récemment la thèse de Sabah Aboussalam sur La pauvreté urbaine 
et les comportements résidentiels à Marrakech. Ce travail de direction de thèses a été plus limité en Algérie, par exemple la thèse de Benjelid, aujourd'hui professeur à Oran, sur le réseau urbain de la péninsule oranaise ;

4 - dans une seconde phase, j'ai assumé un rôle de conseiller pour les équipes de recherche, ce qui ne se confond pas avec un rôle de directeur que je n'ai jamais eu. En Tunisie j'ai eu ce rôle de 1972 à 1976, dans une collaboration avec le Ceres (Centre d'études et de recherches économiques et sociales) de Tunis. Par exemple, j'ai contribué à monter un grand programme de recherche sur le bilan de vingt ans de recherche régionale, initialement avec le Cnrs, auquel ont contribué aussi des non-universitaires. J'ai fait la même chose au Maroc avec plusieurs équipes de recherche de l'Inau (Institut national d'aménagement et d'urbanisme). En Algérie, il m'est arrivé deux fois d'avoir ce rôle au département de Géographie et d'Aménagement de l'université d'Oran ;

5 - dans une troisième phase, je m'insère dans un groupe déjà formé d'une université du Maghreb avec laquelle je travaille, pour un échange d'égal à égal, pour apporter un poids supplémentaire à un département déjà constitué... C'est ce que je fais à l'Enau (École nationale d'aménagement et d'urbanisme) de Tunis, qui a créé il y a six ans un département d'urbanisme habilité au troisième cycle. Son directeur m'a demandé d'organiser une collaboration qui concerne le Dess et le Dea, en complétant la palette des professeurs tunisiens dans plusieurs domaines : j'interviens sur le système urbain et régional, d'autres le font sur le droit de l'urbanisme, etc. L'idée reste que cette collaboration est amenée à disparaitre au fil du temps, avec toujours ce principe de la «formation des formateurs ». De ce fait, il n'y a déjà plus de Français dans le Dess qui a six ans, alors qu'il y en a encore cinq ou six dans le Dea, qui a trois ans. Je n'ai pas d'activité de ce type en Algérie ni au Maroc.

6 Si on revient au début de cette activité... pourquoi a-t-on fait appel à toi, alors que tu travaillais plutôt sur la France et le Brésil ?

7 De façon assez classique dans le milieu universitaire, parce que j'avais déjà une réputation de spécialiste des problèmes économiques et urbains après ma thèse et ma nomination à la Sorbonne en 1964, et aussi dans la logique d'une "relève des générations » (j'ai pris le relais de Pierre George en Tunisie, de Jean Dresch en Tunisie et au Maroc...) auprès des premiers Tunisiens responsables.

8 En France aussi, tu as suivi de nombreux chercheurs du Maghreb en thèse.

9 Ça découle du même processus. Je commençais aussi dans les années 1960 à avoir une timide ouverture sur le tiers-monde dans mes propres recherches puisque je travaillais déjà sur le Brésil. Ces chercheurs sont venus me solliciter parce que je proposais des problématiques nouvelles... et aussi parce que je pouvais accepter des problématiques nouvelles.

10 Que penses-tu du relais qui s'opère actuellement entre la coopération bilatérale et la coopération euro-méditerranéenne avec des programmes comme Med-Campus, des initiatives comme celles du Grerbam?

11 Personnellement j'en suis resté aux échanges bi-latéraux : depuis que je suis en retraite, j'ai été co-initiateur avec le département d'urbanisme de l'Enau de Tunis d'une " commission mixte de coopération universitaire ». En ce qui concerne les échanges euro-méditerranéens, j'ai eu un début d'expérience sur un projet monté par le Politecnico de Milan avec l'Iedes sur les problèmes urbains, mais cette collaboration n'a pas abouti. Au Maroc, le relais a été pris par d'autres sur ces programmes européens. 
12 Dans les années 1950, tu as écrit un article sur l'Italie. Comment, à travers ton expérience de chercheur, analyses-tu la différenciation qui s'est creusée entre les deux rives sur le plan du développement, certains problèmes de sous-développement régional (le Sud italien...) et d'éventuels points communs entre les deux rives comme par exemple l'informel?

13 Je ne suis pas spécialiste de la rive nord, mon impression c'est qu'une région sousdéveloppée dans un pays dont l'ensemble est développé ne pose jamais les mêmes problèmes urbains que ceux des pays dits «sous-développés" ou «en développement». Il y a bien sûr des convergences comme l'informel, mais la problématique $\mathrm{du}$ sous-développement relève fondamentalement de l'échelle nationale, et il ne faut pas surestimer les apparentes convergences. Pour la rive sud, c'est d'abord une réalité de structures, qui est inséparable de la domination coloniale ou post-coloniale... Un clivage régional, si fort soit-il, même s'il relève d'une unification tardive et d'une exploitation sociale, est différent dans sa nature. Au Brésil ce type de clivage, comme entre le Sudeste et le Nordeste, n'a pas le même impact en termes de problèmes urbains que l'histoire coloniale et post-coloniale du Brésil.

\section{AUTEUR}

\section{DOMINIQUE RIVIÈRE}

Maître de conférence à l'université Paris-Val-de-Marne 